\title{
Anaesthesia changes perceived finger width but not finger length
}

Lee D. Walsh ${ }^{1,2}$, Damon Hoad ${ }^{3}$, John C. Rothwell ${ }^{3}$, Simon C. Gandevia ${ }^{2}$ and Patrick Haggard ${ }^{1}$

1. Institute of Cognitive Neuroscience, University College London, London, United Kingdom

2. Neuroscience Research Australia and University of New South Wales, Sydney, NSW, Australia.

3. Sobell Department of Motor Neuroscience and Movement Disorders, Institute of Neurology, University College London, Queen Square, London, UK

Keywords: anaesthesia, hand, body size, body image

Length: 5265 words

Corresponding Author: Dr. Lee Walsh

Neuroscience Research Australia

Margarete Ainsworth Building

Randwick, NW 2031

Australia

Tel: +6129399 1887

Fax: +6129399 1027

E-mail: 1.walsh@neura.edu.au 


\begin{abstract}
The brain needs information about the size of the body to control our interactions with the environment. No receptor signals this information directly; the brain must determine body size from multiple sensory inputs, and then store this information. This process is poorly understood, but somatosensory information is thought to play a role. In particular, anaesthetizing a body part has been reported to make it feel bigger. Here, we report the first study to measure if changes in body size following anaesthesia are uniform across dimensions (e.g. width and length).

We blocked the digital nerves of 10 human subjects with a clinical dose of local anaesthetic (1\% lignocaine $)$ and again in separate sessions with a weaker dose $(0.25 \%$ lignocaine $)$ and a saline control. Subjects reported the perceived size of their index finger by selecting templates from a set that varied in size and aspect ratio. We also measured changes in sensory signals that might contribute to the anaesthetic-induced changes using quantitative sensory testing.

Subjects perceived their finger to be up to $32 \%$ wider during anaesthesia when compared to during a saline control condition. However, changes in perceived length of the finger were much smaller $(<5 \%)$. Previous studies have shown a change in perceived body size with anaesthesia, but have assumed that the aspect ratio is preserved. Our data shows that this is not the case. We suggest that non-uniform changes in perceived body size might be due to the brain increasing the body's perimeter to protect it from further injury.
\end{abstract}




\section{Introduction}

The ability to perceive and move our limbs depends on both direct sensory information and on stored information. Information about body size is one example of critical information that is centrally stored. To determine limb position the brain requires information about the angles of the joints and the length of the limb segments. Proprioceptive information is provided by receptors in the muscle, skin and joint, and also by centrally-generated command signals (see Proske and Gandevia 2012). However, there are no receptors to signal body size, which must be determined indirectly and stored. Furthermore, although planning and executing movement requires the brain to have some information about body size, this information does not need to be accessible to conscious perception or explicit judgement. It has been shown that perceptual judgements about hand shape and size are profoundly distorted (Longo and Haggard 2010), despite our ability for fine control of the digits. This suggests dissociation between information about body part size for motor control and that used for perception. Furthermore, even unconscious body size information is still labile and must be regularly updated (Mon-Williams and Bingham 2007).

How information about body size is determined is not yet clear, but experience obtained during both development (e.g. Ishak et al. 2014) and adulthood (Franchak and Adolph 2014) will be important. These centrally stored body representations are poorly understood but most scientists agree that they are generated from multiple sensory signals. Some proposed mechanisms are based in the parietal areas of the brain involved in multisensory processing (Freund 2003; Blanke and Arzy 2005; Ehrsson et al. 2005; Lewis 2006) and others use wider cortical networks (Schwoebel and Coslett 2005; Tsakiris et al. 2007).

Body representations are surprisingly malleable (e.g. Botvinick and Cohen 1998; Gandevia and Phegan 1999; Paqueron et al. 2003; Gandevia et al. 2006; Walsh et al. 2010; Inui et al. 2011). In particular anaesthesia is known to make body parts feel larger (Gandevia and Phegan 1999; Paqueron et al. 2003; Paqueron et al. 2004a; Inui et al. 2011). However, it is not known if changes in perceived body part size involve equal changes in perceived length and width of the body, maintaining a constant perceived shape of the body part. Anaesthesia may affect perceived shape of the body part as well as its size. Recent studies have shown that perceived shape of individual body parts (Longo and Haggard 2010), and of the whole body (Fuentes et al. 2013) is distorted. A combined investigation of shape and size during anaesthesia is needed to determine if central processing of spatial information is uniform. 
Why the perceived size of the body changes following anaesthesia is not clear. One possibility is that the brain assumes an injury has occurred when sensory information is lost and a perceived increase in size acts to protect the body from further injury. Body parts that feel larger than they are will be kept further away from objects in the environment.

Changes in perceived size of the body have been studied using anaesthesia induced by an ischemic pressure block (e.g. Inui et al. 2011), or by injection of lignocaine (e.g. Gandevia and Phegan 1999; Paqueron et al. 2003). The ischemic pressure block acts slowly and affects the larger diameter afferents that signal touch and proprioception, first. In contrast, local anaesthetics such as lignocaine have the opposite effect, blocking small-diameter afferents first (Gasser and Erlanger 1929; Catterall and Mackie 1996; Paqueron et al. 2004a). It is clear that selectively blocking large diameter afferents alters perceived body size (Inui et al. 2011) and the same has been said about small diameter fibres (Paqueron et al. 2004a). However, the effect of selectively blocking small fibres has not been tested. Measuring body size after selectively removing input from small-diameter fibres, but leaving large fibres intact, will determine the role of smaller diameter afferents in perceiving body size and determine if this role differs from that of large diameter afferent signals.

This study was designed with two aims. First, we aimed to determine whether local anaesthesia produced a perceived change in shape, as well as a change in size, of the finger. Second we attempted to selectively block small-diameter afferents in the digital nerves of the index finger. We therefore measured perceived finger width and length before and after digital nerve block, partial nerve block and injection of saline. We also measured sensory detection thresholds for touch, heat, cold and pain to determine which diameter axons were affected by our partial anaesthesia technique.

\section{Materials and Methods}

Ten healthy subjects ( 7 male) aged 22-37 participated in three experimental sessions. The data for one subject was excluded from session two because clinically complete anaesthesia was not achieved. All subjects gave informed written consent before participating and the University College London Research Ethics Committee approved this study. The experiment was conducted according to the Declaration of Helsinki. The experimental protocol was fully explained to subjects but they were not aware of the experimental hypothesis. Subjects knew when they had been anaesthetised, because the feeling of anaesthesia versus saline are clear, but subjects were naïve to the effects were investigating The authors were not subjects in the study. 


\section{Experimental setup and measurements}

Subjects were seated at a table and their right hand and forearm were placed under a box, so that the distal half of the subject's forearm and their hand were not visible. The hand remained under the box in this way for the whole experimental session, only being removed to administer injections of anaesthetic or saline (see below). We developed a series of measurements to test the subjects' perception of the size and shape of their hands, and we used established quantitative sensory testing measures to quantify the effects of the anaesthetic on sensory afferents. This set of measurements was performed together at several times in all sessions in the order they are described here.

To assess changes in perceived finger size, a template chart was designed using an outline of the lateral side of a right index finger (Fig. 1). This outline was scaled to produce 49 different finger outlines, corresponding to the combination of seven equally spaced widths and seven equally spaced lengths. So for each width all seven lengths were represented giving the 49 differently scaled finger outlines. The smallest undistorted finger template was $22 \mathrm{~mm}$ long and $7 \mathrm{~mm}$ wide at the base. The largest undistorted finger template was $90 \mathrm{~mm}$ long and $26 \mathrm{~mm}$ wide at the base. These outlines were randomly distributed on an A2 sized (594 x $420 \mathrm{~mm}$ ) chart and randomly labelled with the numbers 1 to 49 . Five different template charts were created, each with a different random arrangement of the finger outlines. Two further sets of charts were made in the same way. One showed outlines of only the proximal half of the index finger and the other set showed outlines of only the distal half of the index finger (Fig. 1). If anaesthesia was deeper closer to the injection site, changes in perceived size may differ between the distal and proximal finger. When measurements were taken the subject was first shown a full finger chart and told to "Tell me which finger represents the size of your right index finger." The subject then examined the chart, chose one of the 49 fingers, and reported the corresponding number. The subject was previously instructed, at the start of the session, to always look at the whole chart before answering. Two more charts, selected at random, from the same full finger set were shown in the same way, providing three measurements for the time point. These three measurements were later averaged and the mean was used for analysis. In the same way the subject was then shown three charts from the set showing the proximal half of the finger. Here the instruction was "Tell me which finger represents the size of the base of your right index finger." Following that, the same occurred with the distal finger charts with the instruction "Tell me which finger represents the size of the tip of your right index finger." 
Monofilament detection thresholds were measured separately on distal and proximal parts of the index finger (Fig. 1). A set of calibrated monofilaments using the Semmes Weinstein scale was used. Testing started with the 2.83 monofilament and each filament was pressed on the skin five times at pseudo-random time intervals of less than 3 seconds. If the subject detected three or more of the five touches of the monofilament, detection was taken as successful and the experimenter tried the next thinner monofilament. If detection failed the experimenter tried the next thicker monofilament. After detection of a monofilament had failed twice, the subject's threshold was recorded as the thinnest monofilament that was detected.

Next a thermode was used to measure the threshold for warm and cold sensation. The starting temperature of the thermode was an estimate of skin temperature. This was determined by beginning with atmospheric temperature inside the box covering the subject's hand. The thermode was set to this temperature and held against the subject's skin on the lateral side of the proximal segment of the index finger for $2 \mathrm{~s}$. The subject was asked, "Does the thermode feel warm, cold or neutral." If the subject did not report "neutral" the thermode temperature was adjusted and the test repeated until a "neutral" response from the subject was achieved, this was the starting temperature. Once the starting temperature was established the subject's heat detection threshold was measured. The experimenter told the subject "Tell me when you feel warm". The thermode was placed on the subject's skin and held for at least $3 \mathrm{~s}$ before the temperature was ramped up at a rate of $0.5^{\circ} \mathrm{Cs}^{-1}$. The thermode remained stationary during this period. When the subject indicated that they felt warmth under the thermode, it was removed from the skin and the temperature recorded. Three measurements were taken at each time point and both the distal and proximal sites used for monofilament testing were tested separately (Fig. 1). The same technique was used to measure the subject's cold detection threshold, except that the temperature was ramped down at a rate of $0.5^{\circ} \mathrm{Cs}^{-1}$ and the instruction to the subject was “Tell me when you feel cold". For both measurements of heat and cold the subject was instructed beforehand to report their first sensation of heat or cold, not their tolerance.

Pain threshold to mechanical compression was measured separately at both the distal and proximal site. Here an electronic compression gauge (PFI200N, Mecmesin, Sussex, United Kingdom; range 1-200 N, resolution $0.04 \mathrm{~N}$ ) was used. The gauge had a steel tip that was tubular with an $8 \mathrm{~mm}$ external diameter and a $6 \mathrm{~mm}$ internal diameter. The subject was instructed to indicate the first painful sensation, not their tolerance of the pain. The experimenter said "Tell me when you detect pain" and the open end of the tip of the compression gauge was pressed into the subject's finger until they indicated pain. The force at which the subject 
indicated pain was recorded. Three measurements were taken at each time point and averaged for analysis. The rate of increase of the force applied to the subject's finger by the compression gauge probe was not precisely controlled, but care was taken by the experimenter to produce similar profile across trials and subjects. The experimenter was trained to produce a linear force ramp of $40 \mathrm{~N}$ over $5 \mathrm{~s}$. The same experimenter operated the compression gauge in all trials for all subjects.

\section{Session one - partial anaesthesia}

Subjects were set up in the experimental apparatus and a set of baseline measurements was taken (see above). Then, a total of 3-4 $\mathrm{ml}$ of $0.25 \%$ lignocaine without adrenaline was injected into the medial and lateral side of the subject's right index finger. These injections were made approximately $10 \mathrm{~mm}$ distal to the metacarpophalageal joint and targeted the digital nerves of the finger aiming to partially anaesthetize them. A third injection of $0.5 \mathrm{ml}$ of $1 \%$ lignocaine was injected under the skin of the dorsum of the finger about $10 \mathrm{~mm}$ distal to the metacarpophalageal joint. The purpose of this last injection was to block the superficial radial nerve. This branch of the superficial radial nerve innervates the proximal dorsum of the finger just beyond the metacarpophalageal joint, but how much of this area it innervates varies significantly between people. Although the sites of our sensory testing were outside the field of the superficial radial, in this session it was blocked completely so that its contribution could be excluded and anaesthesia of the digital nerves studied more precisely. After all three injections were complete, a piece of tape was placed around the finger just distal to the metacarpophalageal joint to slightly impede the venous return from the finger and prolong the anaesthesia.

We waited five minutes after completion of the injections to allow the anaesthetic to take effect. During this time the subject's finger was tested continuously for light touch using cotton wool to confirm that the anaesthetic was having some effect. After those five minutes the first set of experimental measurements were taken. Immediately afterwards, a second set of measurements was taken so that we could judge if the partial anaesthesia was stable over our experimental time frame. The time taken to complete each set of measurements was 10 minutes. After the session the tape was removed from the subject's index finger and sensation recovered within a couple of hours.

\section{Session two - complete anaesthesia}

This session was a repeat of the first, with the same subjects, the same measures and the same design. The difference was that all three injections were all done with $1 \%$ lignocaine without 
adrenaline. As this protocol for digital anaesthesia has been used and documented by us previously (e.g. Walsh et al. 2011a; Walsh et al. 2011b), we were confident that it would be stable over the experimental time, and we thus collected only one set of measurements after the injections.

The subject was placed in the apparatus and a baseline set of measurements was taken. Then the anaesthetic was injected using the same procedure and the same three injection sites as the first session. Again, a piece of tape was used to slightly impede venous return in the blocked finger. The injection volumes for each subject were matched to those from the first session so that the swelling of the proximal part of the finger due to the injection volume would be similar. After injection of the anaesthetic we waited until anaesthesia of the index finger was clinically complete, i.e. detection of light touch was abolished. Then the set of experimental measurements was taken. After the session the tape was removed from the finger and the subject recovered within a few hours.

\section{Session three - injection of saline}

Sessions one and two were designed to investigate the effect of local anaesthesia on the perception of width and length of the index finger. However, injection of local anaesthetic near the metacarpophalageal joint causes swelling of the proximal segment of the finger and therefore real changes in the diameter of this part of the finger were expected. This swelling was measured by taking photographs of the dorsum of the subject's finger before and after injection. Measurements of finger width around the injection sites were taken from these photographs to assess the swelling caused by the injections. To control for the effect of this swelling on perceived finger size, we repeated the protocol with saline injections in the same subjects. Session three was performed in the same way as session two except that all three injections contained physiological saline only. The injection volumes for each subject were matched to their injection volumes from sessions one and two. As for session two, baseline measurements were taken before injection and one set of post-injection measurements was taken, because the effect of the saline is stable. There was no need to wait for onset of anaesthesia in this session. However, without anaesthetic some subjects experienced mild pain during the injections. We waited for any pain to subside before the experimental measurements began, generally no more than 5 minutes. A piece of tape was used to slightly impede venous return for consistency with experiments one and two. At the end of the session the tape was removed from the subject's finger and they recovered from the swelling within 30 minutes. 


\section{Data and statistical analysis}

Data was collated and analysed using Igor Pro v6.31 (Wavemetrics, Lake Oswego, OR, USA) and statistical testing was performed with SPSS v20.0.0 (IBM Corp. Armonk, NY, USA). All measurements, except monofilament detection thresholds, were performed three times at each time point and the mean of these three measurements was used for analysis. We were most interested in the change in our measures due to anaesthetic. We calculated the difference between the pre-injection baseline value and the post-injection value for each measure and subject. In the case of the saline and the complete anaesthesia this simply meant subtracting the baseline value for that subject from their value after the injections. In the case of the partial anaesthesia session, we found no significant differences between the two post-injection time points on any measure, and we therefore averaged values at these time-points.

Statistical inference was based on two-way repeated measures ANOVA. Each of the measurements of finger width and length were independent variables in separate ANOVA. In both cases the factors were dose of anaesthetic (saline, partial, or complete) and the factor segment (distal, proximal, full). The factors were the same for the four quantitative sensory tests but the segment factor only had two levels (distal and proximal). When an ANOVA result was significant, pairwise pot-hoc testing was performed so that significant differences between individual doses could be identified. Correction was not performed for multiple comparisons because factors contained three levels (see Cardinal and Aitken 2006). Threshold for significance was $\alpha=0.05$.

\section{Results}

\section{Changes in perceived finger size}

Mean data for all subjects for the three levels of anaesthesia are shown in Figure 2. Subjects reported perceiving a wider finger after anaesthesia, but no change is seen for the perceived length of the finger. The 95\% confidence intervals in Figure 2 show that with complete anaesthesia there is a larger increase in the perceived width of the index finger, but again no change in the perceived length. For individual subjects we calculated a change following anaesthesia by subtracting their perceived finger size before injection from their perceived finger size after injection. Similar calculations were done for sensory detection thresholds. The preinjection means were not significantly different from each other in all but one case, cold detection on the distal finger. Here the baseline detection threshold for saline was significantly smaller than the baseline for complete anaesthesia by $3.4[1.1,5.7]^{\circ}$ (mean $[95 \%$ confidence 
interval]). This was not considered a problem because this was a small difference and the changes in cold detection threshold following saline injection were smaller and non-significant. The baseline measurements of full finger size, proximal finger size and distal finger size did not differ significantly. Changes in sensory detection thresholds between distal and proximal sites were mostly non-significant; in three cases there were significant changes that were too small to explain the differences in distal and proximal measurements. The injections of saline and anaesthetic produced a physical swelling of the proximal segment of the index finger. However, the change in diameter of this part of the finger was only $\sim 4 \%$ on average and non-significant (before $23[21.5,24.5] \mathrm{mm}$, after $24[22.3,25.7] \mathrm{mm}, t_{16.9}=-0.99, p=0.99$ ).

Figure 3 shows the changes in perceived width and length of the index finger. ANOVA showed that the dose of anaesthetic significantly affected the perceived width of the full finger $\left(F_{2,16}=\right.$ $5.59, p=0.014)$. Complete anaesthesia produced a mean change of $29.9[19.9,39.9] \%$ in the perceived width of the index finger. This was about triple the mean change following partial anaesthesia $(9.9[2.8,16.6] \%)$ or saline $(9.5[-0.2,19.2] \%)$ and significantly different from both (Table 1). The perceived change in the length of the whole finger showed no consistent trend (Fig. 3), nor was the main effect of dose significant $\left(F_{2,16}=0.31, p=0.74\right)$.

When subjects were instructed to judge the size of only the proximal half of the index finger the change following complete anaesthesia was similar to that for the full finger $(32.0[18.4,45.6] \%$, Fig. 3), but more variable. The changes following partial anaesthesia $(14.3[-0.60,29.2] \%)$ and saline $(15.7[-1.1,32.5] \%)$ were also more variable and the effect of dose on perceived width was non-significant $\left(F_{2,16}=1.44, p=0.266\right)$. Perceived length of the finger did not change by more than $3.0[-6.7,12.7] \%$ across the three doses of anaesthetic (Fig. 3) and these changes were nonsignificant $\left(F_{2,16}=0.111, p=0.896\right)$.

In the distal half of the finger we found a significant effect of anaesthetic dose $\left(F_{2,16}=4.71, p=\right.$ 0.025) on perceived width of the finger. With complete anaesthesia, subjects reported a mean change in their perceived finger width of $21.1[6.8,35.4] \%$. This change differed significantly from the smaller change following partial anaesthesia $(10.8[0.6,21.0] \%)$ and the negligible change in the saline condition $(1.9[-10.4,14.2] \%)$. Results for the perceived length of the distal half of the index finger were similar to those for the proximal finger with no significant effect of the dose of anaesthetic $\left(F_{2,16}=0.792, p=0.470\right)$.

In summary, our results show that perceived width of the finger was increased with anaesthesia, but changes in perceived length were small or absent. Furthermore, changes of perceived finger 
width after injection of anaesthetic were larger than those seen with only injection of saline. These results show that the change in perceived size following anaesthesia is not uniform, as previously assumed. Rather, we found that the finger is perceived to get up to $30 \%$ fatter, but less than $3 \%$ longer.

\section{Changes in sensory detection}

The results of our quantitative sensory testing are shown in Figure 4 and the results of the ANOVA and pairwise post-hoc tests are shown in Table 2. Generally injection of saline had little or no effect on the detection of our sensory stimuli. The one possible exception is the detection of touch on the proximal part of the finger. Here the monofilament detection threshold increased by $10.4[1.3,19.5] \%$ following the injection of saline (Fig. 4, $t_{9}=63.3, p<0.001$ ). This might be due to the saline applying enough pressure to the nerve to cause a slight block. All four sensory tests showed a change in threshold with injection of anaesthetic. ANOVA showed main effects of dose for all tests in both the proximal and distal part of the finger (Table 2). In general, post-hoc testing showed that the three conditions produced changes in threshold that were significantly different from each other. One exception was the detection of cold in the distal finger, where the difference between the saline and partial anaesthesia conditions was nonsignificant. Pain threshold testing also had mixed post-hoc results, although there was still a significant effect of anaesthetic dose on pain threshold.

The purpose of the quantitative sensory testing was to determine if our partial anaesthesia technique selectively blocked smaller fibres. Our results show this is not the case. Of particular importance are the monofilament detection threshold and the cold detection threshold. These two tests should be mostly selective for large and small myelinated axons, respectively. Small unmyelineated fibres mostly signal pain and heat. The post-hoc analysis for these two sensory tests shows significant differences between the saline and partial anaesthesia conditions (Table 2), similar to the other tests. Thus while our results show that we achieved a stable state of partial anaesthesia, they also show that all afferent populations were affected more or less uniformly.

\section{Discussion}

The key novel findings are, first that the perceived width of the finger changes after anaesthesia but perceived length barely changes, if at all. We are the first to investigate if perceived changes in body size during local anaesthesia are uniform across body dimensions (e.g. width and length). Second, stable partial anaesthesia of the digital nerves is possible, but not as selective for small 
diameter axons as is expected from previous reports (Gasser and Erlanger 1929; Catterall and Mackie 1996; Paqueron et al. 2003; Paqueron et al. 2004a; Paqueron et al. 2004b).

Complete clinical anaesthesia (1\% lignocaine) produced the largest changes in perceived finger width. Partial digital anaesthesia (0.25\% lignocaine) showed some small effects on the perceived width of the index finger (Fig. 3) that were consistent with previous work that showed perceived size changing monotonically with the degree of anaesthesia (Inui et al. 2011). The effects of anaesthesia were larger in the proximal part of the finger than the distal part. This is expected because nerves running to the tip of the finger are expected to be deeper in the nerve trunk and less anaesthetic will diffuse into the centre of the trunk. Thus, the proximal part of the finger will experience deeper anaesthesia than the distal part. Injection of fluid into the base of the finger induces a real change in body size, so a saline control was important to distinguish effects of anaesthesia from effects of the injections. Injection of saline affected the perceived width of the full finger and the proximal half of the finger. Partial anaesthesia had similar effects to saline in the proximal part of the finger, suggesting that the effect was due to swelling, rather than anaesthesia. Comparable effects of saline and partial anaesthesia were also seen when subjects judged the full finger size. However, in the distal half of the finger, where there was no swelling or effect of saline, partial anaesthesia significantly increased perceived finger width. Together these results suggest that partial anaesthesia has modest effects on perception, but care is needed to distinguish these effects from the effects of injecting fluid. It also suggests that the swelling at the base of the finger strongly influences perception of the size of the full finger and that subjects were able to dissociate the size of the distal finger from the size of the proximal finger. Following complete anaesthesia the perceived width of the distal part of the finger increased significantly by $21 \%$ but changes in the perceived length were $<2 \%$ and non-significant. There were no real changes in the size of this part of the finger. The large changes in perceived width with only small changes in perceived length show that these two aspects of body information may be processed and perceived differently.

The length of limb segments is critical for skilled motor actions and it is generally fixed, not changing over time. Even changes in limb length during adolescence are slow ( $0.08-0.1$ $\mathrm{m} /$ annum, Tanner 1962; Visser et al. 1998). Perceived width of the fingers is important for manipulation of objects. Studies of reaching and grasping show careful regulation of grip aperture during reaching (Jeannerod 1981), which is lost following deafferentation of the hand (Jeannerod et al. 1984) or during prosthetic use (Wing and Fraser 1983). Swelling, changes in muscle mass and fat content can produce quite large changes in the width of body segments over 
short time frames. To be useful, a representation of the body needs to be updated, at least intermittently. The different behaviour of perceived width and length following anaesthesia may be related to the likelihood of them changing rapidly.

The changes in perceived body size following anaesthesia might just be side effects of physiological mechanisms such as those discussed above, but such changes could also be beneficial. An increase in the perceived size of finger, hand or arm could be a mechanism to prevent injury; adding a safety margin to keep the body farther from possible sources of injury. The downside of this strategy is that it would deteriorate sensorimotor control because the representation of the body is inaccurate. Figure 5 shows the average change in perceived finger perimeter following complete digital anaesthesia. There appears to be a constant spatial margin around the normal finger boundary. This interpretation requires caution given that changes in perceived length were non-significant. Unfortunately statistical power was not high enough for meaningful post-hoc testing of the spatial margin. Furthermore, we have assumed that the change in width is radially symmetrical, which may not be the case. Separate experiments designed specifically to test these hypotheses are needed. Alternatively, a perceived increase in the body's margin could be due to the skin, or another tissue, being perceived as thicker.

Interestingly, studies of the representation of hand landmarks suggested that the stored representation of the hand is wider and shorter than it really is (Longo and Haggard 2010). Our template matching technique did not show the same distortions in baseline measurements. This inconsistency between landmarks and template-matching methods has been noted before (Longo \& Haggard, 2010). It remains unclear why the brain may maintain two separate representations of the same body parts.

Our data shows that withdrawal of sensory information produces an illusion that body width has changed suggesting that the brain uses afferent information to generate our sense of body size. Others have suggested that rapid cortical reorganization may be the mechanism involved. In animal experiments the removal of afferent input from a digit is followed rapidly by cortical neurons that previously had receptive fields confined to said digit becoming responsive to stimulation of adjacent body parts (Calford and Tweedale 1991). This cortical reorganization is unlikely to be the sole cause of the size illusion because anaesthesia of large body areas also induces a change in perceived size (e.g. Paqueron et al. 2003; Inui et al. 2011). Furthermore, after amputation the human somatosensory system continues to process information from sectioned peripheral nerves that have lost their cutaneous territory (Schady et al. 1994). Cortical 
reorganization could simply involve an unmasking of existing pathways as primary inputs fall silent.

Alternatively, perceived changes in body size may be related to removal of inhibitory background activity at several levels in the somatosensory pathway. In rats, cortical cells become more excitable and spontaneous activity in somatosensory cortex increases following section of the sciatic nerve (Dykes and Lamour 1988). Applying the GABA antagonist bicuculline in cat somatosensory cortex leads to an increase in receptive field size (Alloway et al. 1989). Interestingly, injections of lignocaine into the dorsal column nuclei also produced large increases in receptive field size, but these were driven primarily by increases in the length, rather than the width of the receptive field (Dykes and Craig 1998). However, these mechanisms of local plasticity cannot fully explain the changes in body part size that we observed, for two reasons. First, anaesthesia of an entire limb results in changes in its perceived size (e.g. Paqueron et al. 2003; Inui et al. 2011). Second, if perceived body part size were driven simply by receptive field enlargement, then perceived length should increase as much as perceived width, or even more. This is not what we observed. One possibility is that changes in the perceived size of the finger arise at a secondary level of body representation, which exhibits a different organization not based on receptive fields.

A second aim of this study was to induce stable partial anaesthesia of the digital nerves of the index finger by selectively blocking small-diameter fibres and leaving most large diameter fibres intact. Large-diameter nerve fibres are responsible for signalling discriminative touch and joint movement. Small myelinated fibres mostly signal cold and the small unmyelinated fibres mostly signal heat and pain. We were successful in achieving stable partial anaesthesia of the digital nerves that was repeatable across subjects, but we were unsuccessful in selectively blocking small fibres (see Fig. 4). The development of a selective technique would have allowed us to consider the changes in perceived body size after small fibres have been removed, but large fibres are intact. Previous work by Paqueron and colleagues (2003) showed that small fibres are more readily blocked than large fibres. They further suggested that removal of small-fibre input was an important factor in the development of the illusion of increase body size. In contrast more recent work has shown that during an ischemic pressure block, changes in perceived body size begin when large fibres have been blocked but smaller axons remain mostly intact (Inui et al. 2011). Our data is unable to shed any light on this issue.

We blocked the digital nerves, which are smaller than the brachial plexus trunks blocked by Paqueron and colleagues. A smaller nerve bundle requires less anaesthetic, and diffusion into the 
centre of the bundle will be faster. We found no evidence for a temporal progression of anaesthesia in our quantitative sensory testing data. The small size of the digital nerves may have ensured a high concentration of anaesthetic around the nerve, so that large diameter axons were blocked very shortly after small fibres. Lower doses may achieve selective blocking in the digital nerves, but our pilot work suggested that the effects would not be repeatable across subjects. Selective partial block remains an interesting possibility for large nerves.

In summary, we show for the first time that the changes in perceived body size following anaesthesia are not uniform. Changes in perceived width were large, but changes in perceived length were much smaller or absent. This suggests that the brain processes information about the width of a body part differently to the way it processes information about its length. The net effect may be to add a constant margin to the finger's perimeter, perhaps to prevent injury. Representations of body size may include body boundary, which is also vital to movement control. In addition, a stable partial anaesthetic block of the digital nerves is possible but does not selectively block small fibres. Our results are important for understanding how the brain generates and stores representations of body size and suggesting possible functions of these representations.

\section{Acknowledgements}

This work was funded by the National Health and Medical Research Council (of Australia), the EU FP7 Project VERE Work Package 1 and the European Research Council Advanced Grant HUMVOL. The authors declare no competing financial interests.

\section{Conflict of interest}

The authors declare that they have no conflict of interest.

\section{Ethical approval}

All procedures performed in studies involving human participants were in accordance with the ethical standards of the institutional and/or national research committee and with the 1964 Helsinki declaration and its later amendments or comparable ethical standards.

\section{Informed consent}

Informed consent was obtained from all individual participants included in the study. 


\section{References}

Alloway KD, Rosenthal P, Burton H (1989) Quantitative measurements of receptive field changes during antagonism of GABAergic transmission in primary somatosensory cortex of cats. Exp Brain Res 78:514-532

Blanke O, Arzy S (2005) The Out-of-Body Experience: Disturbed Self-Processing at the Temporo-Parietal Junction. The Neuroscientist 11:16-24

Botvinick M, Cohen J (1998) Rubber hands 'feel' touch that eyes see. Nature 391:756

Calford MB, Tweedale R (1991) Acute changes in cutaneous receptive fields in primary somatosensory cortex after digit denervation in adult flying fox. J Neurophysiol 65:178187

Cardinal RN, Aitken MRF (2006) ANOVA for the behavioural sciences researcher. Lawrence Erlbaum Associates, New Jersey, USA

Catterall W, Mackie K (1996) Local anesthetics. In: Hardman JGG, Gilman A, Limbird LL (eds) Goodman and Gilman's The Pharmalogical Basis of Therapeutics, 9th edn. McGraw Hill, New York; Sydney, pp 331-347

Dykes RW, Craig AD (1998) Control of size and excitability of mechanosensory receptive fields in dorsal column nuclei by homolateral dorsal horn neurons. J Neurophysiol 80:120-129

Dykes RW, Lamour Y (1988) An electrophysiological laminar analysis of single somatosensory neurons in partially deafferented rat hindlimb granular cortex subsequent to transection of the sciatic nerve. Brain Res 449:1-17

Ehrsson HH, Holmes NP, Passingham RE (2005) Touching a rubber hand: feeling of body ownership is associated with activity in multisensory brain areas. J Neurosci 25:1056410573

Franchak JM, Adolph KE (2014) Gut estimates: Pregnant women adapt to changing possibilities for squeezing through doorways. Atten Percept Psychophys 76:460-472

Freund H (2003) Somatosensory and motor disturbances in patients with parietal lobe lesions. Adv Neurol 93:179-193

Fuentes CT, Longo MR, Haggard P (2013) Body image distortions in healthy adults. Acta Psychol 144:344-351

Gandevia SC, Phegan CML (1999) Perceptual distortions of the human body image produced by local anaesthesia, pain and cutaneous stimulation. J Physiol 514:609-616

Gandevia SC, Smith JL, Crawford M, Proske U, Taylor JL (2006) Motor commands contribute to human position sense. J Physiol 571:703-710

Gasser HS, Erlanger J (1929) The role of fiber size in the establishment of a nerve block by pressure or cocaine. Am J Physiol 88:581-591

Inui N, Walsh LD, Taylor JL, Gandevia SC (2011) Dynamic changes in the perceived posture of the hand during ischaemic anaesthesia of the arm. J Physiol 589:5775-5784

Ishak S, Franchak JM, Adolph KE (2014) Perception-action development from infants to adults: perceiving affordances for reaching through openings. J Exp Child Psychol 117:92-105

Jeannerod M (1981) Intersegmental coordination during reaching and natural visual objects. In: Long J, Baddeley A (eds) Attention and Performance IX. Lawrence Erlbaum, Hillsdale, NJ, pp 153-168

Jeannerod M, Michel F, Prablanc C (1984) The control of hand movements in a case of hemianaesthesia following a parietal lesion. Brain 107:899-920

Lewis JW (2006) Cortical Networks Related to Human Use of Tools. The Neuroscientist 12:211231

Longo MR, Haggard P (2010) An implicit body representation underlying human position sense. Proc Natl Acad Sci USA 107:11727-11732 
Mon-Williams M, Bingham GP (2007) Calibrating reach distance to visual targets. J Exp Psychol Human 33, 645-656

Paqueron X, Gentili ME, Willer JC, Coriat P, Riou B (2004a) Time sequence of sensory changes after upper extremity block: swelling sensation is an early and accurate predictor of success. Anesthesiology 101:162-168

Paqueron X, Leguen M, Gentili ME, Riou B, Coriat P, Willer JC (2004b) Influence of sensory and proprioceptive impairment on the development of phantom limb syndrome during regional anesthesia. Anesthesiology 100:979-986

Paqueron X, Leguen M, Rosenthal D, Coriat P, Willer JC, Danziger N (2003) The phenomenology of body image distortions induced by regional anaesthesia. Brain 126:702-712

Proske U, Gandevia SC (2012) The proprioceptive senses: Their roles in signalling body shape, body position and movement and muscle force. Physiol Rev 92:1651-1697

Schady W, Braune S, Watson S, Torebjörk HE, Schmidt R (1994) Responsiveness of the somatosensory system after nerve injury and amputation in the human hand. Ann Neurol 36:68-75

Schwoebel J, Coslett HB (2005) Evidence for Multiple, Distinct Representations of the Human Body. J Cogn Neurosci 17:543-553

Tanner JM (1962) Growth at Adolescence (2nd Ed.). Blackwell Scientific Publications, Oxford

Tsakiris M, Hesse MD, Boy C, Haggard P, Fink GR (2007) Neural signatures of body ownership: a sensory network for bodily self-consciousness. Cereb Cortex 17:2235-2244

Visser J, Geuze RH, Kalverboer AF (1998) The relationship between physical growth, the level of activity and the development of motor skills in adolescence: Differences between children with DCD and controls. Hum Movement Sci 17:573-608

Walsh LD, Gandevia SC, Taylor JL (2010) Illusory movements of a phantom hand grade with the duration and magnitude of motor commands. J Physiol 588:1269-1280

Walsh LD, Moseley GL, Taylor JL, Gandevia SC (2011a) Proprioceptive signals contribute to the sense of body ownership. J Physiol 589:3009-3021

Walsh LD, Taylor JL, Gandevia SC (2011b) Overestimation of force during matching of externally generated forces. J Physiol 589:547-557

Wing AM, Fraser C (1983) The contribution of the thumb to reaching movements. Q J Exp Psychol A 35:297-309 


\begin{tabular}{|c|c|c|}
\hline Finger segement & Dose pair & Post-hoc \\
\hline \multirow[t]{3}{*}{ Full finger } & Saline v Partial & $p=0.894$ \\
\hline & Saline v Complete & $p=0.018$ \\
\hline & Partial v Complete & $p=0.045$ \\
\hline \multirow[t]{3}{*}{ Proximal finger } & Saline v Partial & No main \\
\hline & Saline v Complete & effect \\
\hline & Partial v Complete & of dose \\
\hline \multirow[t]{3}{*}{ Distal finger } & Saline v Partial & $p=0.470$ \\
\hline & Saline v Complete & $p=0.019$ \\
\hline & Partial v Complete & $p=0.078$ \\
\hline
\end{tabular}

Table 1: Post-hoc comparisons of the effect of anaesthetic dose on perceived finger width.

Bold text indicates a $p$-value below the threshold of $\alpha=0.05$. 


\begin{tabular}{|c|c|c|c|c|}
\hline Sensory test & Finger segment & Main effect & Dose pair & Post-hoc \\
\hline \multirow[t]{6}{*}{ Monofilament } & \multirow[t]{2}{*}{ Proximal finger } & \multirow[t]{2}{*}{$F_{2,16}=37.5, p<0.001$} & Saline v Partial & $p=0.029$ \\
\hline & & & Saline v Complete & $p<0.001$ \\
\hline & \multirow{4}{*}{ Distal finger } & \multirow{4}{*}{$F_{2,16}=9.72, p=0.002$} & Partial v Complete & $p=0.001$ \\
\hline & & & Saline v Partial & $p=0.040$ \\
\hline & & & Saline v Complete & $p=0.011$ \\
\hline & & & Partial v Complete & $p=0.018$ \\
\hline \multirow[t]{5}{*}{ Cold } & \multirow[t]{3}{*}{ Proximal finger } & \multirow[t]{2}{*}{$F_{2,16}=26.2, p<0.001$} & Saline v Partial & $p=0.011$ \\
\hline & & & Saline v Complete & $p<0.001$ \\
\hline & & & $\begin{array}{l}\text { Partial v Complete } \\
\text { Saline v Partial }\end{array}$ & $\begin{array}{l}\boldsymbol{p}=\mathbf{0 . 0 0 8} \\
p=0.079\end{array}$ \\
\hline & \multirow{2}{*}{ Distal finger } & \multirow{2}{*}{$F_{2,16}=11.7, p=0.001$} & Saline v Complete & $p=0.004$ \\
\hline & & & Partial v Complete & $p=0.009$ \\
\hline \multirow[t]{5}{*}{ Heat } & \multirow[t]{2}{*}{ Proximal finger } & \multirow[t]{2}{*}{$F_{2,16}=27.2, p<0.001$} & Saline v Partial & $p=0.002$ \\
\hline & & & Saline v Complete & $p=0.001$ \\
\hline & \multirow{3}{*}{ Distal finger } & \multirow{3}{*}{$F_{2,16}=8.58, p=0.003$} & $\begin{array}{l}\text { Partial v Complete } \\
\text { Saline v Partial }\end{array}$ & $\begin{array}{l}p=0.001 \\
p=0.047\end{array}$ \\
\hline & & & Saline v Complete & $p=0.013$ \\
\hline & & & Partial v Complete & $p=0.028$ \\
\hline \multirow{6}{*}{$\begin{array}{l}\text { Pain to } \\
\text { compression }\end{array}$} & \multirow[t]{2}{*}{ Proximal finger } & \multirow[t]{3}{*}{$F_{2,16}=8.53, p=0.003$} & Saline v Partial & $p=0.430$ \\
\hline & & & Saline v Complete & $p=0.025$ \\
\hline & \multirow{4}{*}{ Distal finger } & & Partial v Complete & $p=0.009$ \\
\hline & & \multirow{3}{*}{$F_{2,16}=4.32, p=0.032$} & Saline v Partial & $p=0.439$ \\
\hline & & & Saline v Complete & $p=0.069$ \\
\hline & & & Partial v Complete & $p=0.071$ \\
\hline
\end{tabular}

Table 2: ANOVA and pairwise post-hoc results for the Quantitative Sensory Testing

measures. The four sensory tests were performed on both the proximal and distal half of each finger. ANOVA was used to test for a main effect of the dose of anaesthetic and the pairwise post-hoc tests followed to determine differences between the three individual doses. Bold text in the 'Main effect' and 'Post-hoc' column indicate $p$-values below $\alpha=0.05$. 


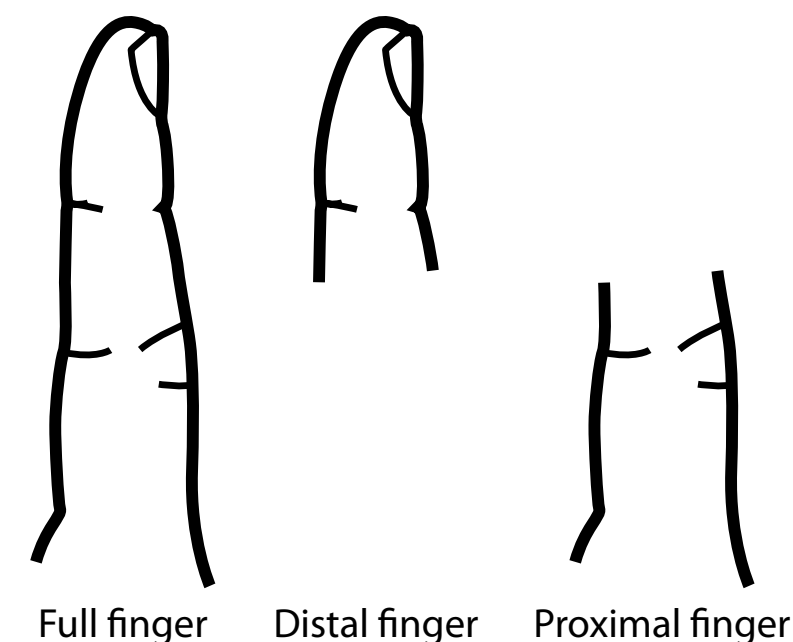

Figure 1: Examples of the templates used to report size. These templates were scaled independently in width and length over seven sizes to generate 49 templates. Templates were randomly arranged on an A2 sheet and randomly labelled with numbers from 1 to 49 . Five different charts were generated for each of the three templates shown here. 


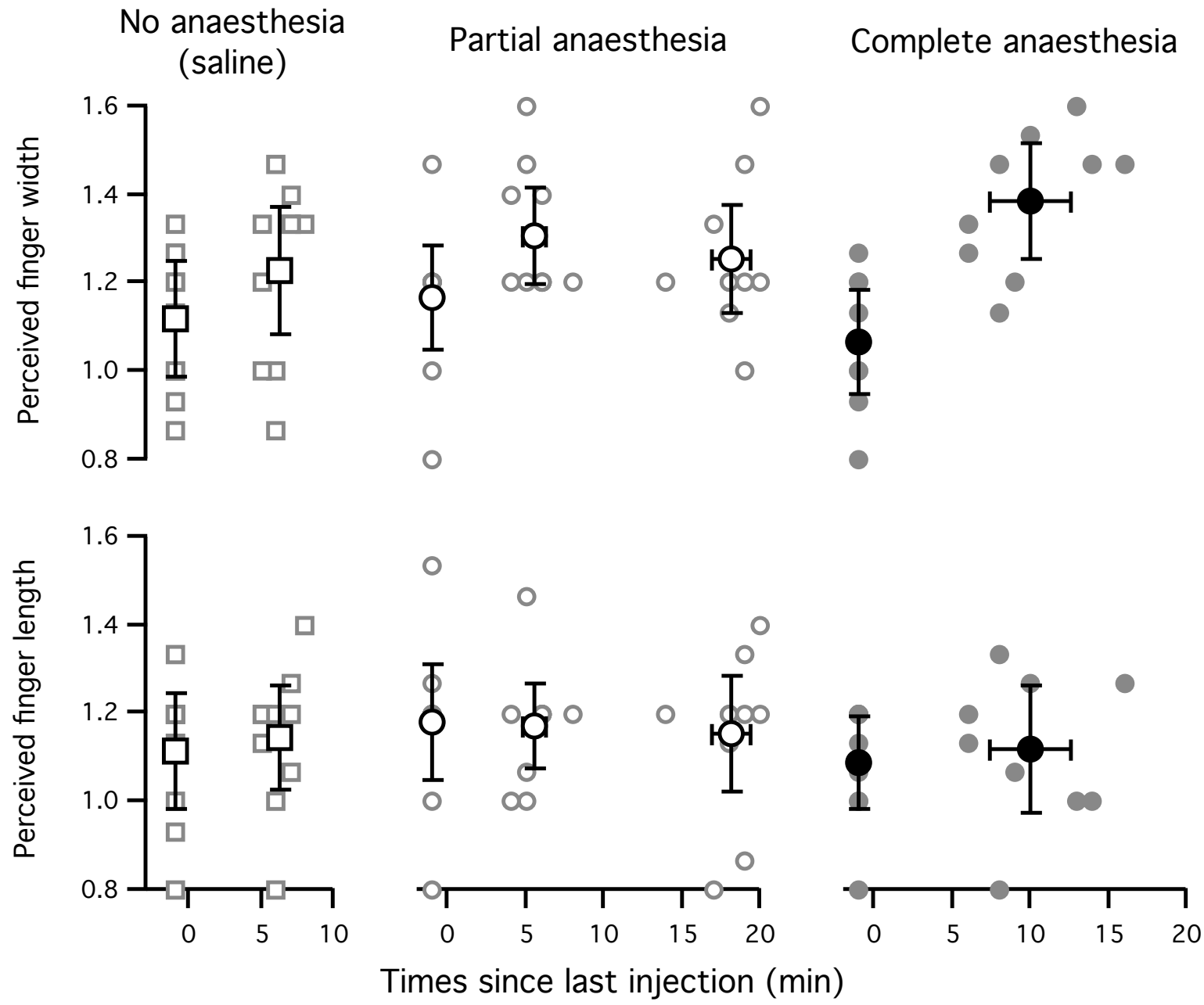

Figure 2: Group and individual subject means ( $\pm 95 \%$ confidence interval) of the perceived width and length for the index finger before and after digital nerve anaesthesia. Columns from left to right show the effect of saline (open squares), partial anaesthesia $(0.25 \%$ lignocaine, open circles) and clinically complete anaesthesia (1\% lignocaine, closed circles) of the digital nerves. The top row shows the perceived width of the index finger and the bottom row shows the perceived finger length. The ordinate scale is relative to a 'standard' finger template, which is represented by the value 1.0. Small grey markers show means for individual subjects and large black markers show the group mean ( \pm 95 confidence interval). Zero on the time axis is when the last injection was complete. Data points before zero show pre-injection data. 

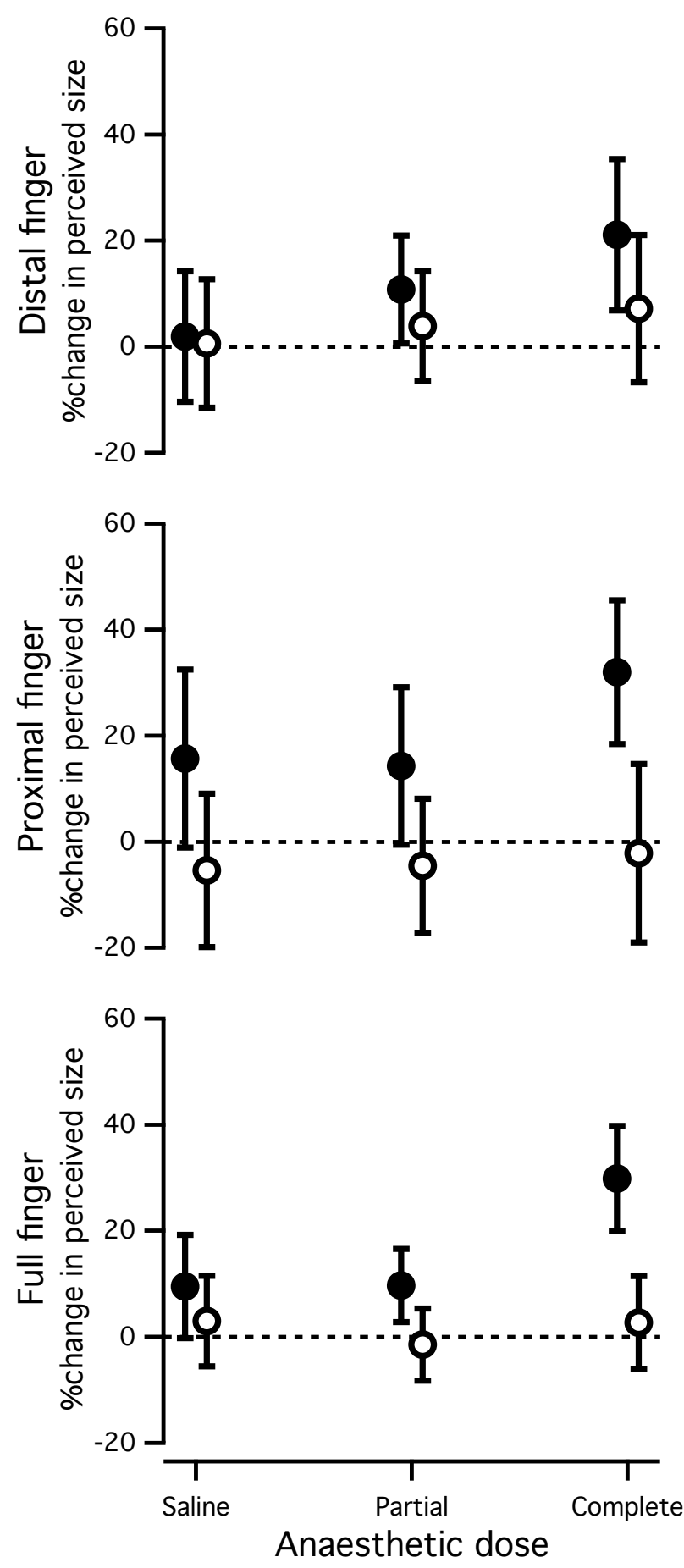

Figure 3: Group mean ( $\pm 95 \%$ confidence interval) percentage change in perceived finger width (closed circles) and length (open circles) for the index finger before and after digital nerve anaesthesia. From top to bottom the rows show the percentage change when judging the distal half, proximal half or full finger. Three doses of lignocaine were used $0 \%$ (saline), $0.25 \%$ (partial) and $1 \%$ (complete). 

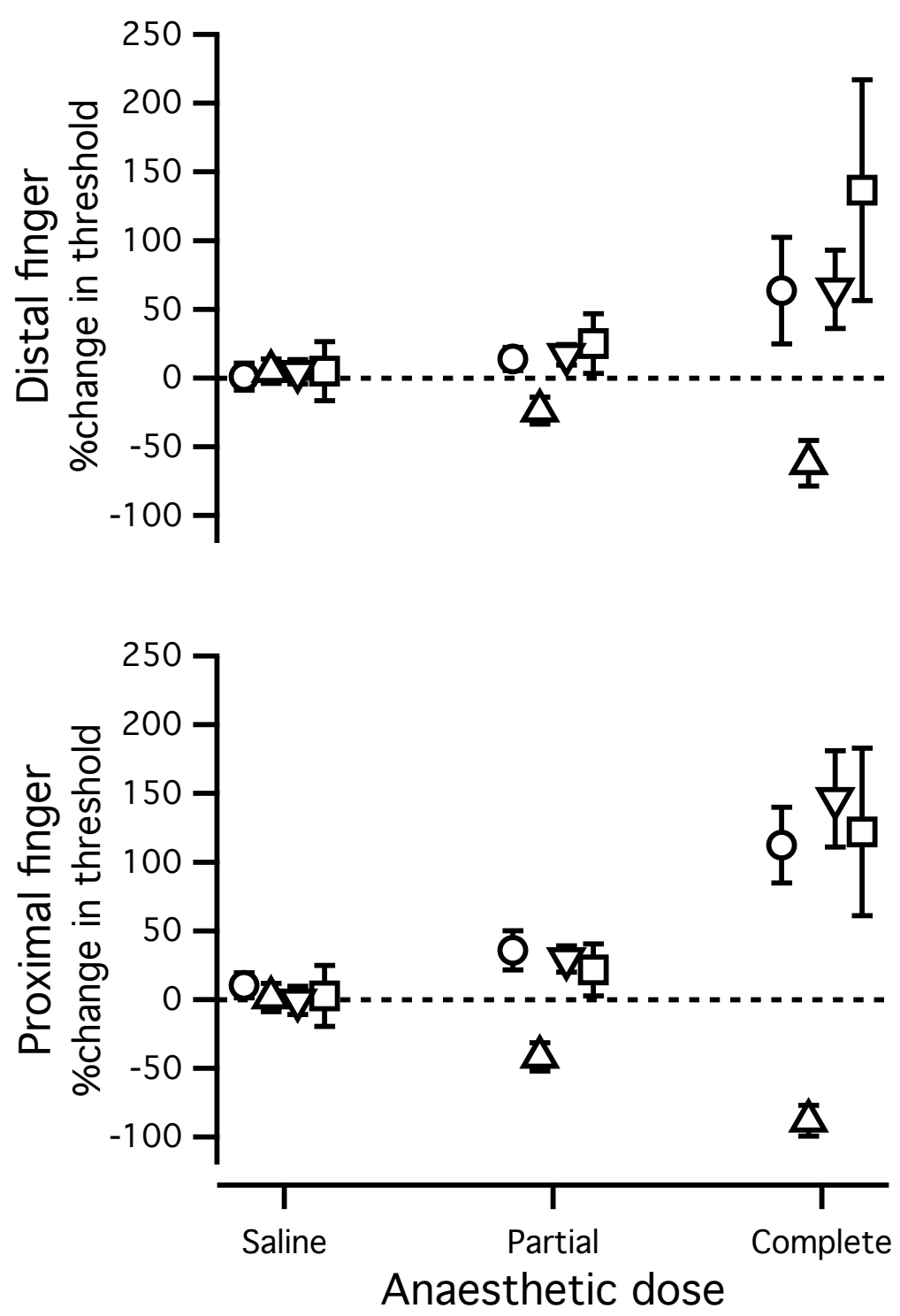

Figure 4: Group mean $( \pm 95 \%$ confidence interval) of the percentage change in qualitative sensory testing measures. Four measures were used, monofilament detection (circles), cold detection (up triangles), heat (down triangle) and pain to compression (squares). The top row shows the percentage change in the effect when judging the distal half of the finger and the bottom row shows the effect of the proximal half of the finger. Three doses of lignocaine were used $0 \%$ (saline), $0.25 \%$ (partial) and $1 \%$ (complete). 


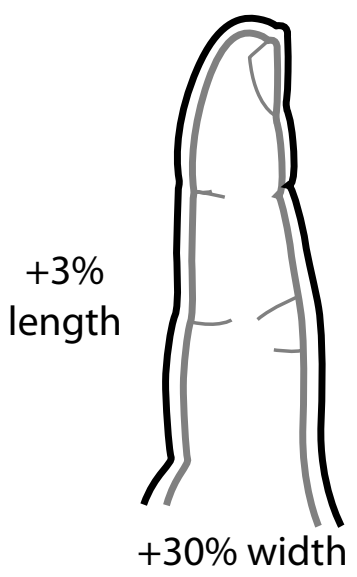

Figure 5: Diagrammatic representation of finger perimeter before (grey line) and after (black line) complete digital anaesthesia. 